Acta Crystallographica Section D

Biological

Crystallography

ISSN 1399-0047

Gergely Róna, ${ }^{\mathrm{a} *}$ Máté Borsos, ${ }^{\mathrm{a}}$

Bostjan Kobe $^{b}$ and Beáta G.

Vértessy ${ }^{\mathrm{a}, \mathrm{c} *}$

anstitute of Enzymology, RCNS, Hungarian Academy of Sciences, 1117 Budapest, Hungary,

${ }^{\mathbf{b}}$ School of Chemistry and Molecular Bio-

sciences, Institute for Molecular Bioscience and Australian Infectious Diseases Research Centre, The University of Queensland, Brisbane, Queensland 4072, Australia, and ${ }^{\mathrm{C}}$ Department of Applied Biotechnology, Budapest University of Technology and Economics, 1111 Budapest, Hungary

Correspondence e-mail: rona.gergely@ttk.mta.hu, vertessy.beata@ttk.mta.hu

Received 27 May 2014

Accepted 9 September 2014

\section{Factors influencing nucleo-cytoplasmic trafficking: which matter? Response to Alvisi \& Jans' comment on Phosphorylation adjacent to the nuclear localization signal of human dUTPase abolishes nuclear import: structural and mechanistic insights}

The authors respond to a comment by Alvisi \& Jans [(2014), Acta Cryst. D70, 2775-2776] on the article Phosphorylation adjacent to the nuclear localization signal of human dUTPase abolishes nuclear import: structural and mechanistic insights [Róna et al. (2013), Acta Cryst. D69, 2495-2505].

Nucleo-cytoplasmic trafficking of proteins is tightly regulated in a number of ways (Poon \& Jans, 2005; Pouton et al., 2007; Sekimoto \& Yoneda, 2012). Post-transcriptional modifications play a key role in these regulatory processes, and phosphorylation can either enhance or reduce nuclear accumulation (Jans, 1995; Nardozzi et al., 2010). Phosphorylation in the vicinity of nuclear localization signals (NLSs) can affect the binding affinity between the cargoes and their nuclear transport receptors. A negative charge in the proximity to the positively charged NLS can diminish its recognition by importin- $\alpha$, while it might have an enhancing effect if positioned further upstream of the NLS (Alvisi et al., 2008; Fontes et al., 2003; Harreman et al., 2004; Hübner et al., 1997; Kosugi et al., 2008, 2009; Marfori et al., 2012). Until now, however, no clear crystallographic model has been available explaining how phosphorylation inhibits cargo protein binding to importin- $\alpha$. The crystallographic model presented in our study has now shed light on a structural mechanism that lies behind the phosphorylation-mediated inhibition of nuclear import (Róna et al., 2013).

Recent studies have shown that the cytoplasmic retention factor BRAP2 can recognize NLSs or NLS-like sequences upon phosphorylation, and therefore can affect the localization pattern of several viral and cellular proteins (Fulcher et al., 2010; Li et al., 1998). However, even overexpressed BRAP2 was not able to completely block its binding partners (either endogenous or also overexpressed) from entering the nucleus, since these binding partners still remained mainly nuclear. BRAP2 therefore appears to have mainly a finetuning function in determining the nuclear levels of its binding partners (Fulcher et al., 2010).

Nevertheless, the involvement of BRAP2 in the cytoplasmic localization of the NLS phosphorylated (S11) dUTPase (Ladner et al., 1996; Róna et al., 2013; Takacs et al., 2009; Tinkelenberg et al., 2003), as suggested by Alvisi \& Jans (2014), is an interesting hypothesis but could only be answered after careful experimental testing. Our unpublished preliminary experiments aiming at identifying the binding partners of human dUTPase did not detect BRAP2 as its binding partner, although further confirmation of this result is needed. Even though the classical monopartite NLS (class 2) (Kosugi et al., 2009) of dUTPase resembles some of the phospho-NLSs BRAP2 binds (ppUL44, T-ag, p53), the phosphorylatable moiety is positioned differently relative to the positively charged cluster.

Alvisi \& Jans (2014) argue that mechanisms other than the phosphorylation affecting binding to importin- $\alpha$ may be responsible for the cytoplasmic localization profile of phosphorylated dUTPase, because the hyperphosphorylation mimicking mutation (S11E) only impairs the $K_{\mathrm{d}}$ value of the dUTPase:importin- $\alpha$ interaction by 
tenfold. They suggest that according to the work of Hodel et al. (2001) and Harreman et al. (2004), only mutations resulting in changes of two orders of magnitude in the affinity constant would have a significant impact on nuclear translocation. Based on the available literature, we are of the opinion that this is a somewhat oversimplified interpretation of the issue. Hodel and coworkers (Hodel et al., 2001) established that functional NLSs have dissociation constants for importin- $\alpha$ binding in the range of $10 \mathrm{n} M$ to $1 \mu M$. This is a rather wide range of values and what most likely matters for a particular NLS is where its affinity lies within this range, rather than the magnitude of the effect of phosphorylation, i.e. whether the phosphorylation moves the affinity over the threshold so it falls outside the functional range. If the affinity of a certain cargo:importin- $\alpha$ interaction is close to the low affinity limit for a functional NLS, a small difference can have a considerable effect. On the other hand, if the $K_{\mathrm{d}}$ value is close to the high affinity limit, a much more substantial alteration of the NLS would be required to make it non-functional.

Alvisi \& Jans (2014) suggest that based on the results of Harreman et al. (2004), a tenfold difference in binding affinity would not by itself explain the strong impact of the S11E mutation on the cellular localization observed in our study (Róna et al., 2013). However, the data in the same study clearly indicates (Harreman et al., 2004) that affinity differences of less than tenfold could lead to drastic changes in the localization of the cargo protein. The wild-type SV40 large Tantigen NLS derivative used in their study (SPKKKRKAE, termed SV40A7) had a $K_{\mathrm{d}}$ value for $\triangle$ IBB-importin- $\alpha$ of $80 \mathrm{n} M$, while its hyperphosphorylation mimicking mutant variant (EPKKKRKAE, termed SV40A7E) had a $K_{\mathrm{d}}$ value of $283 \mathrm{n} M$, as determined in vitro by a GFP anisotropy-based binding assay. The ability of the SV40A7 NLS to drive a GFP reporter construct to the nucleus was validated in vivo in yeast. However, the SV40A7E mutant was not imported into the nucleus, despite the just 3.5-fold affinity difference compared to the wild-type NLS. The NLS of Swi6 was also tested in a similar manner. The wild-type NLS (SPLKKLKID) had a $K_{\mathrm{d}}$ value of $26 \mathrm{n} M$, while the hyperphosphorylation mimicking mutant (EPLKKLKID) had a $K_{\mathrm{d}}$ value of $124 \mathrm{n} M$. The mutant NLS was not able to drive GFP accumulation into the nucleus, although the affinity difference was only 4.8 -fold. The authors also used the full-length sequence of Swi6 fused to GFP to validate their results. The wild-type Swi6 protein had a $K_{\mathrm{d}}$ value of $45 \mathrm{n} M$, while the $K_{\mathrm{d}}$ value of the phosphorylationmimicking mutant was $163 \mathrm{n} M$. The phosphorylation-mimicking mutant was not able to enter the nucleus in vivo, despite only a 3.5fold difference in the $K_{\mathrm{d}}$ value compared to the wild-type Swi6, which was able to enter the nucleus in the G1 phase (Harreman et al., 2004).

We believe that our data are in agreement with previous reports in the literature, and that a tenfold affinity difference between the phosphorylation-mimicking (S11E) and wild-type dUTPase for importin- $\alpha$ could realistically be the sole reason for the nuclear exclusion of the phosphorylation-mimicking protein. However, the data certainly allow the possibility that cytoplasmic retention factors, such as BRAP2, could contribute to the fine-tuning of the localization pattern of dUTPase. This possibility will need to be investigated experimentally.

This work was supported by the Hungarian Scientific Research Fund (OTKA NK 84008, OTKA K109486), the Baross program of the New Hungary Development Plan (3DSTRUCT, OMFB-00266/ 2010 REG-KM-09-1-2009-0050), the Hungarian Academy of Sciences (TTK IF-28/2012 and MedinProt) and the European Commission FP7 BioStruct-X project (contract No. 283570), to BGV. BK is a National Health and Medical Research Council Research Fellow (APP1003325). GR is recipient of Young Researcher Fellowships from the Hungarian Academy of Sciences.

\section{References}

Alvisi, G. \& Jans, D. A. (2014). Acta Cryst. D70, 2775-2776.

Alvisi, G., Rawlinson, S. M., Ghildyal, R., Ripalti, A. \& Jans, D. A. (2008). Biochim. Biophys. Acta, 1784, 213-227.

Fontes, M. R., Teh, T., Toth, G., John, A., Pavo, I., Jans, D. A. \& Kobe, B. (2003). Biochem. J. 375, 339-349.

Fulcher, A. J., Roth, D. M., Fatima, S., Alvisi, G. \& Jans, D. A. (2010). FASEB J. 24, 1454-1466.

Harreman, M. T., Kline, T. M., Milford, H. G., Harben, M. B., Hodel, A. E. \& Corbett, A. H. (2004). J. Biol. Chem. 279, 20613-20621.

Hodel, M. R., Corbett, A. H. \& Hodel, A. E. (2001). J. Biol. Chem. 276, $1317-$ 1325.

Hübner, S., Xiao, C. Y. \& Jans, D. A. (1997). J. Biol. Chem. 272, 17191-17195.

Jans, D. A. (1995). Biochem J. 311, 705-716.

Kosugi, S., Hasebe, M., Entani, T., Takayama, S., Tomita, M. \& Yanagawa, H. (2008). Chem. Biol. 15, 940-949.

Kosugi, S., Hasebe, M., Matsumura, N., Takashima, H., Miyamoto-Sato, E., Tomita, M. \& Yanagawa, H. (2009). J. Biol. Chem. 284, 478-485.

Ladner, R. D., Carr, S. A., Huddleston, M. J., McNulty, D. E. \& Caradonna, S. J. (1996). J. Biol. Chem. 271, 7752-7757.

Li, S., Ku, C. Y., Farmer, A. A., Cong, Y. S., Chen, C. F. \& Lee, W. H. (1998). J. Biol. Chem. 273, 6183-6189.

Marfori, M., Lonhienne, T. G., Forwood, J. K. \& Kobe, B. (2012). Traffic, 13, 532-548.

Nardozzi, J. D., Lott, K. \& Cingolani, G. (2010). Cell. Commun. Signal. 8, 32.

Poon, I. K. \& Jans, D. A. (2005). Traffic, 6, 173-186.

Pouton, C. W., Wagstaff, K. M., Roth, D. M., Moseley, G. W. \& Jans, D. A. (2007). Adv. Drug. Deliv. Rev. 59, 698-717.

Róna, G., Marfori, M., Borsos, M., Scheer, I., Takacs, E., Toth, J., Babos, F., Magyar, A., Erdei, A., Bozoky, Z., Buday, L., Kobe, B. \& Vertessy, B. G. (2013). Acta Cryst. D69, 2495-2505.

Sekimoto, T. \& Yoneda, Y. (2012). Genes Cells, 17, 525-535.

Takacs, E., Barabas, O., Petoukhov, M. V., Svergun, D. I. \& Vertessy, B. G. (2009). FEBS Lett. 583, 865-871.

Tinkelenberg, B. A., Fazzone, W., Lynch, F. J. \& Ladner, R. D. (2003). Exp. Cell Res. 287, 39-46. 